\title{
Passive vibration absorber with dry friction
}

\author{
A. Hartung, H. Schmieg, P. Vielsack
}

Summary The properties of a passive vibration absorber with dry friction significantly differ from those of the classical linear absorber. The exceptional phenomenon is the possibility of suppressing all excited modes. This effect is influenced to a small extent by a special shape of the friction characteristic, but mainly by an appropriately adjusted threshold of the static friction. The theoretical predictions are confirmed by experimental investigations.

Keywords Nonlinear Absorber, Dry Friction, Experiment

1

Introduction

A passive vibration absorber is a mass-spring subsystem coupled to a superstructure to control its oscillations under the action of periodic excitation. A simple form of this arrangement is shown in Fig. 1 where $M_{1}$ is a mass emulating the superstructure and $K_{1}$ is its mounting spring. The second mass, $M_{2}$, the coupling spring $K_{2}$ and a viscous damper $d$ constitute the absorber system. The superstructure is driven by a harmonic base motion with amplitude $A$ and angular frequency $\Omega$. Let $x_{1}$ be the displacement of $M_{1}$ and $x_{2}$ the displacement of $M_{2}$, respectively. So far, the problem is well known from elementary textbooks on linear vibration theory.

Now, a friction device is added to the substructure which turns the problem into a strongly nonlinear mechanical system. The law for the friction force $R$ must be defined in a way that $R$ is an active force if the device slides, and a passive one if the device sticks. This gives strict separation between stick and slip phases during motion.

Classical investigations on motions of mechanical systems with dry friction are mostly based on deterministic laws which are defined by the product of a dynamic friction coefficient, depending on the relative velocity at the contact area, and the normal pressure, generally depending on time, [1]. In the following, the normal force is assumed to be constant during motion. Then, the dynamic friction force can be reduced to a simple expression $R=R_{d} \operatorname{sgn} \dot{x}_{2}+a \dot{x}_{2}$. Introducing the threshold value $R_{s}$ for the static friction force, three possibilities will be investigated as plotted in Fig. 2.

The simplest possibility is Coulomb's law (Fig. 2a). Here, $R_{s}$ is equal to $R_{d}$, and the dynamic force $R$ depends only on the direction of sliding and not on the value of the relative velocity $\dot{x}_{2}$. In the case of a decreasing characteristic (Fig. 2b), the equality $R_{s}=R_{d}$ still holds, but the friction force depends linearly on the relative velocity $\dot{x}_{2}$ with a negative slope $a<0$. In the third case (Fig. 2c), the value $R_{d}$ of dynamic friction remains constant for $\dot{x} \neq 0$, but the static friction coefficient $R_{s}$ is larger than $R_{d}$.

The first question is whether or not different laws lead to significantly different responses and phenomena of the vibration absorber. Secondly, the total behaviour of the mechanical system is of interest, compared with the well-known efficiency of the classical linear vibration absorber. And finally, experimental investigations should confirm the theoretical results.

Received 10 January 2000; accepted for publication 26 September

2000

A. Hartung, H. Schmieg ( $₫)$, P. Vielsack

Institut für Mechanik, Universität Karlsruhe,

D-76128 Karlsruhe, Germany

Fax: (0721) 6087990

E-mail: Mechanik@bau-verm.uni-karlsruhe.de 


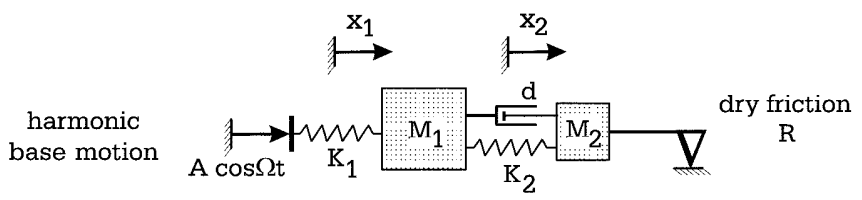

Fig. 1. Mechanical model

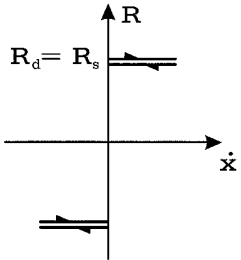

a

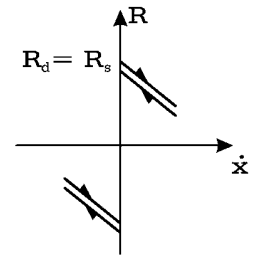

$\mathrm{b}$

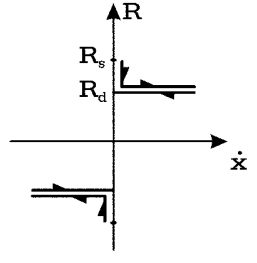

C
Fig. 2a-c. Friction laws. a Coulomb's law, b decreasing characteristic, c static friction larger than dynamic friction

\section{2}

\section{Equation of motion and integration procedure}

Comprehensive literature on the subject of nonsmooth dynamical systems has been made available in the last decade, [2]. The motion of the nonsmooth dynamical system considered can consist of three states at the friction device, i.e. $M_{2}$ slides to the right $\left(\dot{x}_{2}>0\right), M_{2}$ slides to the left $\left(\dot{x}_{2}<0\right)$, and $M_{2}$ sticks $\left(\dot{x}_{2} \equiv 0\right)$. In the last case, the 2-DOF system degenerates into a $1-\mathrm{DOF}$ system, and the active friction force turns over to a passive contact force.

For the computation of the time response, a dimensionless representation of all quantities is recommendable. A dash indicates differentiation with respect to a dimensionless time $\tau=t \sqrt{K_{2} / M_{2}}$. The coordinates $\xi_{i}=x_{i} / A, i=1,2$ are referred to the amplitude $A$ of the excitation. The parameters of the system are given by the friction coefficients $\rho_{s}=R_{s} /\left(A K_{2}\right)$ and $\rho_{d}=R_{d} /\left(A K_{2}\right)$, the mass ratio $m=M_{1} / M_{2}$, the stiffness ratio $k=K_{1} / K_{2}$, the viscous damping coefficient $D=d / \sqrt{K_{2} M_{2}}$ and the slope of the characteristic $D^{*}=a / \sqrt{K_{2} M_{2}}$. Then, the property of the drive is simply given by the excitation frequency ratio $\eta=\Omega \sqrt{M_{2} / K_{2}}$.

In a state of sliding, the equations of motion read

$m \xi_{1}^{\prime \prime}+D \xi_{1}^{\prime}-D \xi_{2}^{\prime}+(1+k) \xi_{1}-\xi_{2}=k \cos (\eta \tau)$,

$\xi_{2}^{\prime \prime}-D \xi_{1}^{\prime}+\left(D+D^{*}\right) \xi_{2}^{\prime}-\xi_{1}+\xi_{2}=-\rho_{d} \operatorname{sgn} \xi_{2}^{\prime}$,

The validity of these equations must be controlled by the condition $\xi_{2}^{\prime} \neq 0$. In the state of sticking, only one equation

$m \xi_{1}^{\prime \prime}+D \xi_{1}^{\prime}+(1+k) \xi_{1}-\xi_{2}^{*}=k \cos (\eta \tau)$

exists. Its validity is controlled by the fact that the passive contact force must be smaller than the threshold value $\rho_{s}$ which reads $\left|\xi_{2}^{*}-\xi_{1}-D \xi_{1}^{\prime}\right|<\rho_{s}$. The constant displacement $\xi_{2}^{*}$ is known from the end of the preceding state of sliding.

The total motion consists of a sequence of intermittent states described by Eqs. (1) or (2). Each state is valid during a certain time interval which depends on the history of motion. The transition points between two successive states are called switching times. They are determined by switching conditions. If the velocity of the friction device in a state of sliding reaches the value

$\xi_{2}^{\prime}=0$,

sliding in one direction is terminated. Sticking is terminated if the condition

$\left|\xi_{2}^{*}-\xi_{1}-D \xi_{1}^{\prime}\right|=\rho_{s}$,

holds.

At the end of each state, there must be a decision about the following state for times larger than the last switching time $\tau_{0}$. The transition is controlled by the switching decision

$\rho\left(\tau_{0}\right)=\xi_{2}^{*}-\xi_{1}\left(\tau_{0}\right)-D \xi_{1}^{\prime}\left(\tau_{0}\right)$, 
which corresponds to the value of the passive contact force at time $\tau_{0}$. If a state of sliding is terminated, two possibilities have to be taken into account: $\left|\rho\left(\tau_{0}\right)\right|<\rho_{s}$ indicates a transition from sliding to sticking, and $\left|\rho\left(\tau_{0}\right)\right|>\rho_{s}$ gives a sudden reversal of the direction of sliding with $\operatorname{sgn} \xi_{2}^{\prime}\left(\tau_{0}+0\right)=-\operatorname{sgn} \xi_{2}^{\prime}\left(\tau_{0}-0\right)$. At the end of a state of sticking, only sliding is possible. The direction of sliding equals the direction of the contact force at $\tau_{0}$, which leads to $\operatorname{sgn} \xi_{2}^{\prime}\left(\tau_{0}+0\right)=\operatorname{sgn} \rho\left(\tau_{0}-0\right)$.

The values of variables $\xi_{1}, \xi_{1}^{\prime}, \xi_{2}, \xi_{2}^{\prime}$ at the end of a certain state give the initial conditions for the equation(s) of motion of the following state. The total solution is pieced together. The process is strongly history-dependent. Procedures for integrating nonsmooth dynamical systems, therefore, consist of two tasks. Firstly, the integration on the linear equations of motion (1) or (2) within two successive switching times. In the case of sticking (Eq. 2), an explicit analytical solution can be given very easily. In the state of sliding, the equations of motion (1) contain unsymmetrical damping. No analytical solution is known. Therefore, a numerical integration of both cases is chosen. This will be done with a Runge-Kutta formula, as described in [3]. This procedure has already been used successfully in [4]. Secondly, the numerical determination of the switching times by considering the switching conditions (3) and (4). Problems arising from this procedure have been discussed in [5] and [6].

\section{3}

\section{Theoretical results}

A numerical calculation needs numbers for the parameters $m, k$ and $D$ of the mechanical system. Classical passive vibration absorbers are designed in a way that $m$ and $k$ are as large as possible, which means that the absorber itself should be a small vibratory subsystem. With view on the experimental investigations, this demand cannot be fulfilled in the present case, because the friction device needs a certain geometrical dimension to ensure safe and reproducible experimental results. Therefore, in the following, the values $m=4$ and $k=1$ are chosen for both numerical and experimental investigations. Viscous damping $D=0.005$ is assumed to be very small. This value agrees with the experimentally measured damping of the experimental arrangement in the case of vanishing dry friction (2-DOF system).

The optimum design of a vibration absorber system is normally discussed by frequency response curves in the case of the classical linear problem. Because the principle of superposition does no longer exist for the nonlinear system with dry friction under consideration, all following results are valid for fixed parameters only. But one can hope that they can be extended in general, at least in a qualitative sense.

The following graphs do not provide any statements on the periodicity or non-periodicity and uniqueness of stationary solutions, [7]. Therefore, the value

$\frac{A_{i}}{A}=\frac{\left(\max \xi_{i}-\min \xi_{i}\right)}{2}, \quad i=1,2$,

is defined to be the amplitude of the response. In the following plots (Figs. 3-5) two limit cases exist: at the top, the frequency response curves for the 2-DOF system without friction showing resonance peaks at $\eta=\eta_{1}$ and $\eta=\eta_{2}$; at the bottom, the frequency response curves for the 1-DOF system with one resonance peak at $\eta=\eta_{0}$, for the case when the friction force tends to infinity. Both graphs, well known from the linear vibration absorber, will serve as a reference in the discussion about the influence of friction within the range $0<\rho_{s}<\infty$.

Figure 3 shows a family of frequency response curves for Coulomb's law. Starting with the linear system without friction (Fig. 3a), first the antiresonance $A_{1} / A=0$ at $\eta=1.0$ disappears even for very low values of $\rho_{s}$ (Fig. 3b). A further increase of $\rho_{s}$ leads to a removal of the resonance peak at the second natural frequency $\eta_{2}$ (Fig. 3c). Exceeding an exciting frequency higher than the second natural frequency $\eta_{2}$, the vibration absorber does no longer move. Within a small increase from $\rho_{s}=0.33$ (Fig. 3d) to $\rho_{s}=0.375$ (Fig. 3e), the resonance peak at the first natural frequency $\eta_{1}$ also vanishes suddenly. From now on, increasing values of $\rho_{s}$ produce standstill of the vibration absorber for a wide range of exciting frequencies. In addition, the resonance peak at the natural frequency $\eta_{0}$ of the 1-DOF system appears. The most interesting phenomenon in the evolution of frequency response curves under consideration is the existence of a certain $\rho_{s}$-range, with small amplitudes, independent of the value of the exciting frequency (Fig. 3e-f). Properly chosen friction can suppress high amplitude motions, which leads to a global stabilization in the 

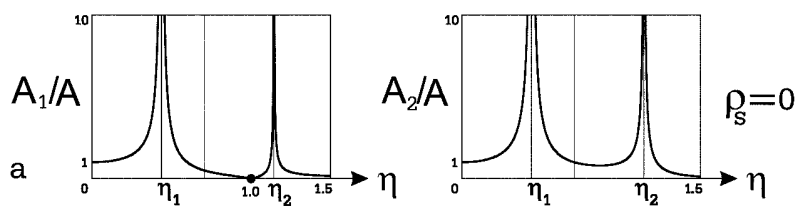

$\mathrm{b}$
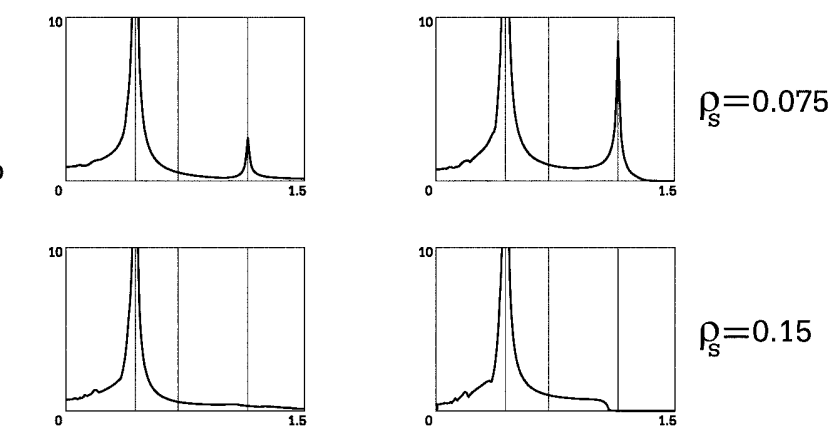

$\rho_{\mathrm{S}}=0.15$
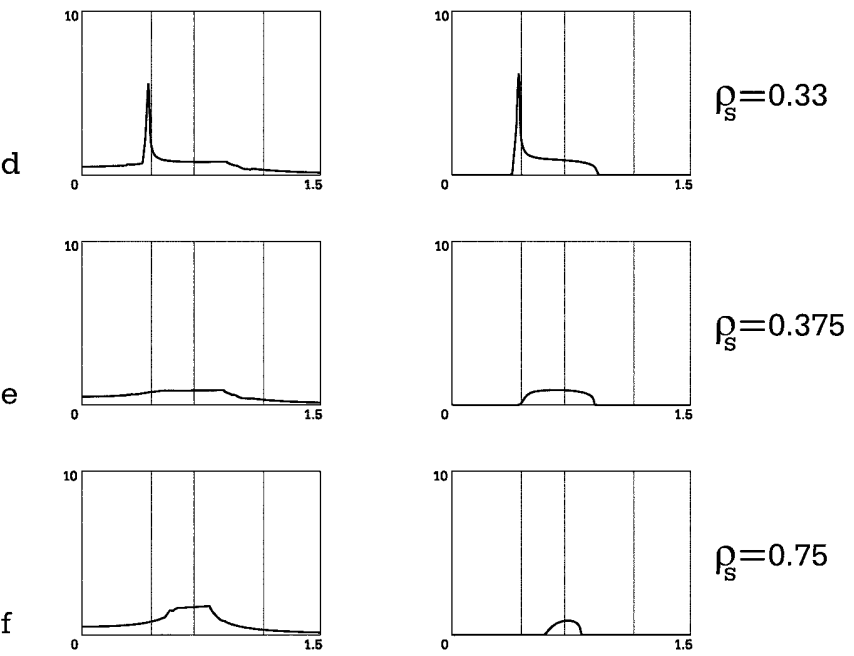

$\rho_{s}=0.75$
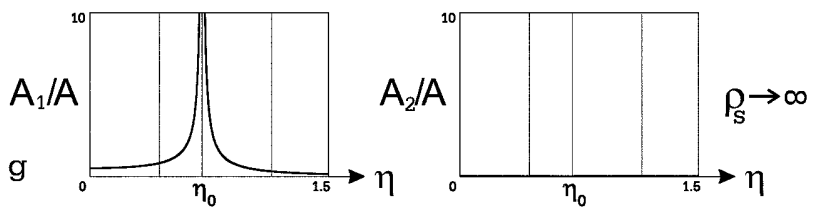

Fig. 3a-g. Frequency response curves for Coulomb friction

entire frequency range. Such a saturation phenomenon is known from active nonlinear absorbers, [8].

Figure 4 shows the corresponding evolution of frequency response curves for the case of a decreasing characteristic. The values $\rho_{s}$ of the static friction are the same as before. The second frictional parameter $D^{*}=-0.04$ is intentionally chosen to be relatively large, to emphasize possible differences in comparison to Coulomb's law. On the other hand, the friction force cannot become too small or even negative in reality. The computation is, therefore, interrupted when the friction force becomes less than half the statical value $\rho_{s}$. This is the case in Fig. $4 \mathrm{~b}$ and $\mathrm{c}$ at the resonance peaks.

Comparing the shape of the family of frequency response curves with the preceding ones shows no qualitative differences between Coulomb friction and a decreasing characteristic. Even the amplitudes are of the same order of magnitude. Quantitatively, there exists a small but negligible additional peak in the vicinity of $\eta_{0}$. The differences caused by both friction laws are insignificant. Even the elimination of the resonance peaks occur for the same values $\rho_{s}$ as before.

The same statements are valid for the third law considered, Fig. 5. Here again, an unrealistic value $\rho_{d}=\rho_{s} / 2$ is chosen to emphasize the difference in comparison to Coulomb's law. The influence of $\rho_{d}$ results in the fact that the suppression of high amplitude motions is shifted to 


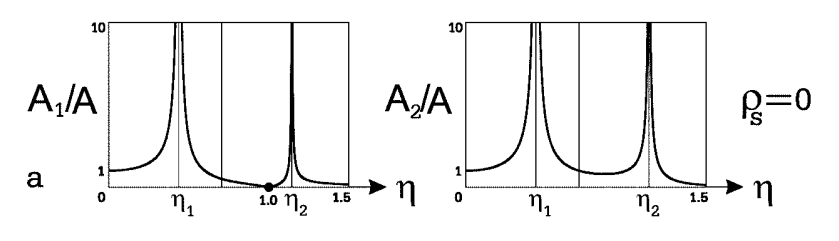

b
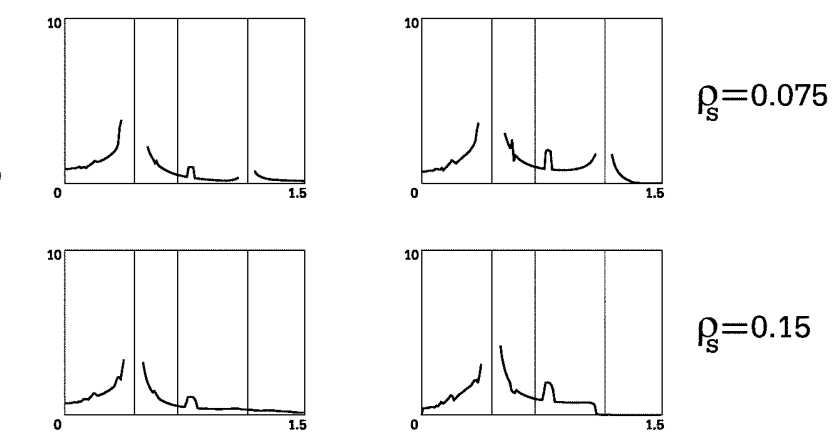

$$
\rho_{S}=0.15
$$

$\mathrm{d}$
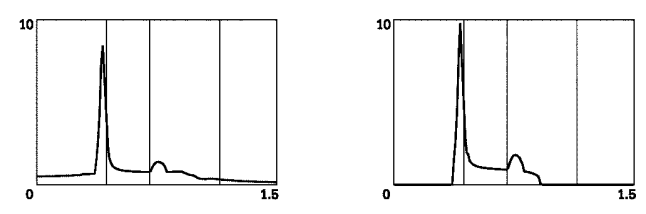

$\rho_{s}=0.33$

e
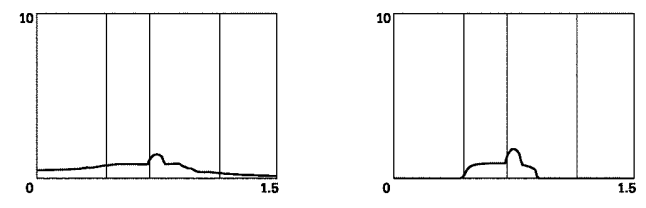

$\rho_{S}=0.375$

$\mathrm{f}$
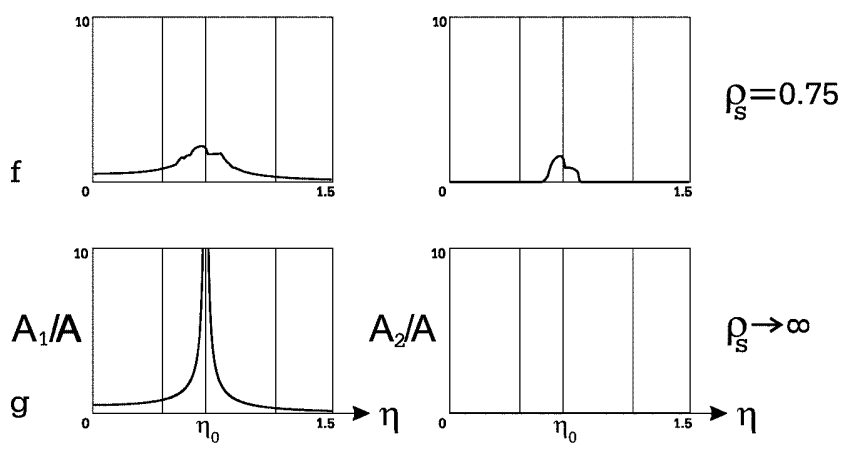

Fig. 4a-g. Frequency response curves for a decreasing characteristic $D^{*}=-0.04$

larger values of $\rho_{s}$, Fig. 5f. If $\rho_{d}$ tends to $\rho_{s}$, the system behaviour tends to the case of Coulomb friction.

Comparing the results for the three different friction laws shows that the system's response is influenced only to a small extent by the special shape of the friction characteristic for velocities $\left|\dot{x}_{2}\right|>0$, but significantly by the static friction coefficient $\rho_{s}$ at $\dot{x}_{2}=0$, which is responsible for a transition from sticking to sliding. A similar statement can be found in [9] for stick-slip motions induced by decelerative sliding.

\section{4}

\section{Experimental investigations}

The aim of the experimental investigation is to confirm the frequency response relationship for different values of $\rho_{s}$, to prove the existence of stick phases and to identify the corresponding friction laws. The latter task causes severe uncertainties because friction laws are influenced not only by mechanical parameters, such as relative velocity and normal force at the contact area, but also by a change of the material properties at the interface, temperature, surface lubrication, wear, etc., [1]. To exclude the above-mentioned effects as much as possible the investigation is restricted to a single frictional material which is used in the engineering practice for pads of car-disc brakes, [10]. 


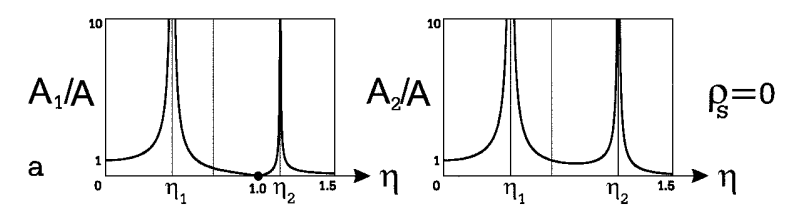

$\mathrm{b}$
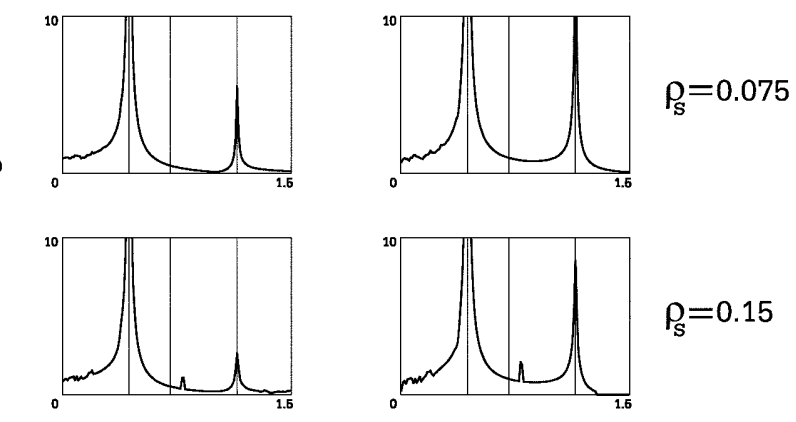

$$
\rho_{\mathrm{S}}=0.15
$$

d
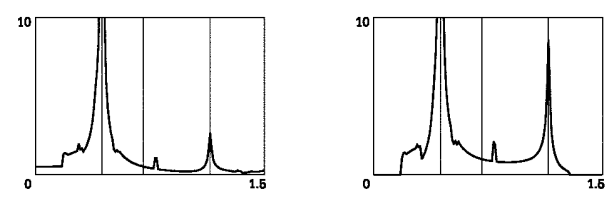

$$
\rho_{\mathrm{s}}=0.33
$$
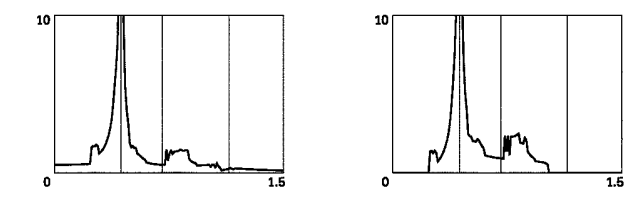

$\rho_{\mathrm{S}}=0.375$
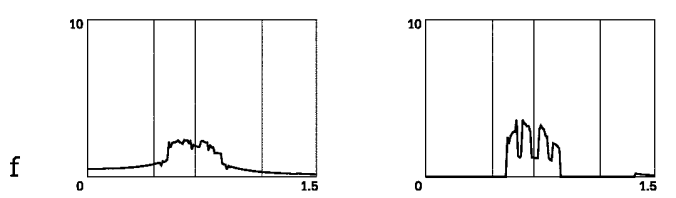

$$
\rho_{\mathrm{S}}=0.75
$$
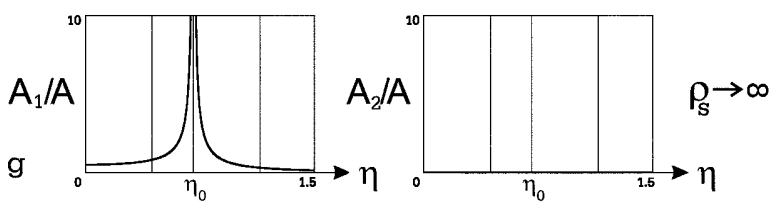

Fig. 5a-g. Frequency response curve for the law $\rho_{d}=\rho_{s} / 2$

\section{1}

\section{Description of the experiments}

The experimental setup consists of the mechanical system with sensors to determine its state and a data acquisition system with a visual programming language, optimized for measurements and also used for the graphical representation of the data. Figure 6 shows the scheme of the mechanical part and the location of the sensors in a qualitative scale.

The mechanical system consists of two masses and two springs. The masses are cylinders of high quality regarding geometry and surface. The cylinder on the left carries two additional masses; its total mass is $M=8.35 \mathrm{~kg}$. The right cylinder is a tube with $M / 4$ corresponding to the theoretical assumption. Both cylinders are mounted in air bearings. This leads to a very small damping coefficient $D=0.003$. The springs are rings made of high-quality steel with a large diameter of $300 \mathrm{~mm}$. They act in both directions and are linear for small deflections compared to the diameter. The linear behaviour was confirmed by means of experiments. A spring constant of $K=0.67 \mathrm{~N} / \mathrm{mm}$ was measured. The harmonic motion of the shaker pin, clamped at one side of the left spring, has a constant amplitude $A=2.5 \mathrm{~mm}$ for all experiments. The other side of this spring is clamped at mass $M$. The right spring connects the mass $M$ with the mass $M / 4$. An adjustable friction member acts on this mass.

Figure 7 shows the scheme of the friction element in detail. The two arms on both sides of the cylinder $M / 4$ are hinged and pressed against the cylinder by a guided pin, movable by a nut. A flat spring is clamped at the left end of the pin, which causes normal forces when the nut 
SIDE VIEW

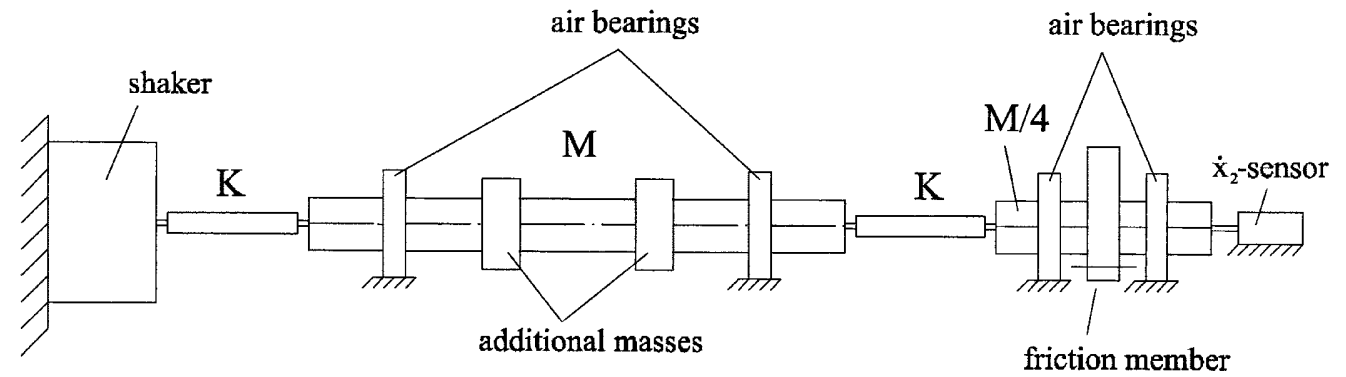

TOP VIEW

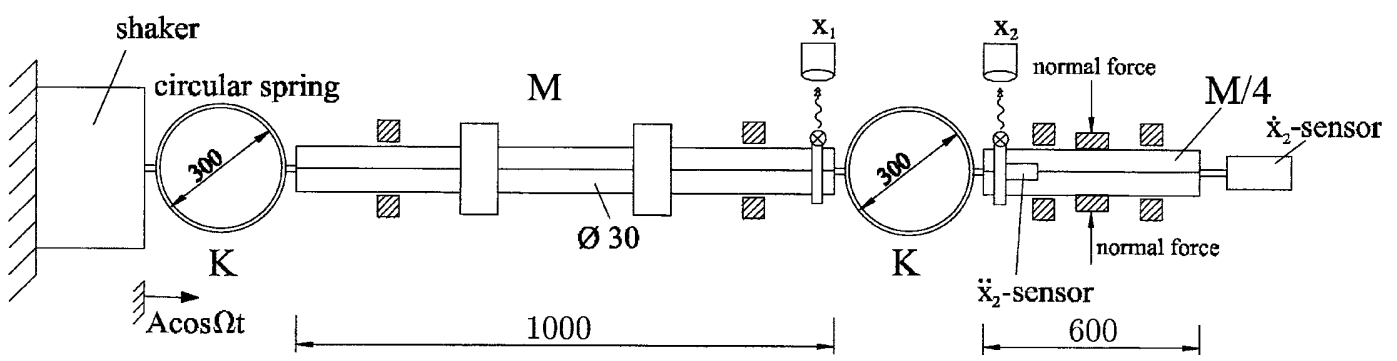

Fig. 6. Scheme of the experimental setup

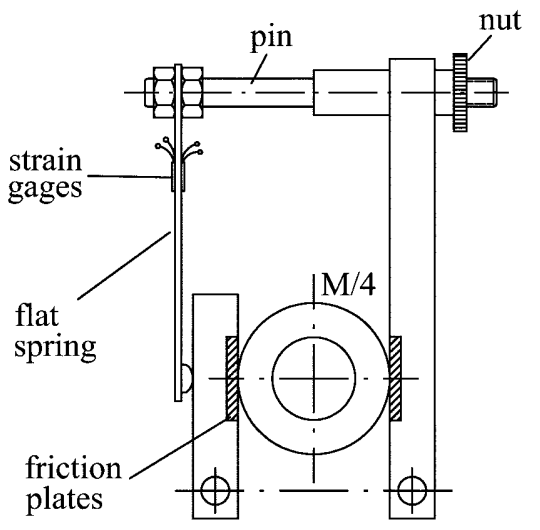

Fig. 7. Scheme of the friction member

is screwed down. Both friction plates are made of pad material of disc brakes and embedded in the arms. The friction between the cylinder and both plates acts along two straight lines.

The normal force is correlated to the signal of strain gages on the flat spring after calibrating its value by separate experiments. This signal allows the reproduction of definite normal forces for repeated measurements. To measure the state of the mechanical system at time $t$, further sensors are necessary. Firstly, opto-electronic position sensors are used for the displacements $x_{1}(t)$ and $x_{2}(t)$ of both masses and an inductive transducer for the motion of the drive. The last signal is needed to control the amplitude $A$ and to measure the angular frequency $\Omega$ of the exciter. The acceleration $\ddot{x}_{2}(t)$ of the mass $M / 4$ is given by an accelerometer. The velocity $\dot{x}_{2}(t)$ is measured by an inductive transducer. A moved permanent magnet in a coil induces a voltage which is strongly proportional to the velocity. It is noteworthy that sticking with $\dot{x}_{2}=0$ can be clearly identified. All signals are digitalized and stored in a computer. The applied program HP VEE allows the combination of different signals.

4.2 Discussion of the results

The theoretical investigations are based on the fact that the normal force only influences the static friction value $R_{s}$. The shape of the characteristic is independent of the normal force. 
The first aim is to identify the friction characteristic $R=R\left(\dot{x}_{2}\right)$ for different normal forces. For this purpose three signals are combined to give the contact force

$-R(t)=\frac{M}{4} \ddot{x}_{2}(t)+K\left(x_{2}(t)-x_{1}(t)\right)$,

depending on time $t$. In addition, $\dot{x}_{2}(t)$ is measured. Figure 8a shows an example of $R(t)$ and Fig. $8 \mathrm{~b}$ of $\dot{x}_{2}(t)$ during about 1.5 periods of excitation with frequency $1.92 \mathrm{~Hz}$. The velocity diagram shows phases of sliding and sticking of approximately the same duration.

The combination of $R(t)$ and $\dot{x}_{2}(t)$ can be interpreted as parametric representation of the friction characteristic $R\left(\dot{x}_{2}\right)$. Eliminating time $t$ leads to the result given in Fig. 9.

All characteristics are based on measurements with the same exciting frequency $f=1.72 \mathrm{~Hz}(\eta=0.6)$, but different normal forces. Additional experiments with a fixed value of the normal force have shown that a change in the frequency does not change the property of a certain characteristic. Figure $9 \mathrm{a}$ fits to Coulomb's law. The nondimensional value of the static threshold is $\rho_{s}=0.55$. A change to a larger value of the normal force gives $\rho_{s}=1.0$, Fig. $9 \mathrm{~b}$. A slight difference between $\rho_{s}$ and $\rho_{d}$ is present. A relatively large normal force, producing $\rho_{s}=4.4$, changes the characteristic significantly. The result resembles the one given in [11]. Obviously, the magnitude of the normal force does not only influence the static threshold $\rho_{s}$, as assumed in theory, but generally the whole characteristic.

The second aim of the experimental investigations is to determine the frequency response curves for a constant amplitude of excitation but varying exciting frequency, keeping the normal force at the friction member fixed. These measurements are performed with a very slow sweep of the exciting frequency, in order to obtain the stationary response of the mechanical system. Because of the natural frequencies $f_{1}=1.121 \mathrm{~Hz}, f_{0}=1.978 \mathrm{~Hz}$ and $f_{2}=3.166 \mathrm{~Hz}$, a range from 0.9 to $3.4 \mathrm{~Hz}$ was chosen with a sweep time of half an hour. The maximum
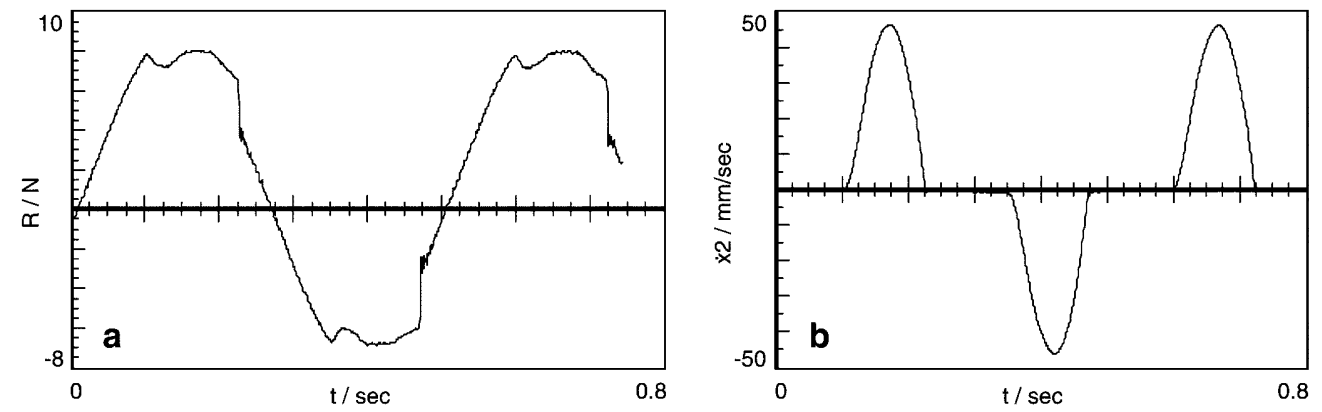

Fig. 8a, b. Stationary oscillation. a Contact force versus time, b velocity $\dot{x}_{2}$ versus time
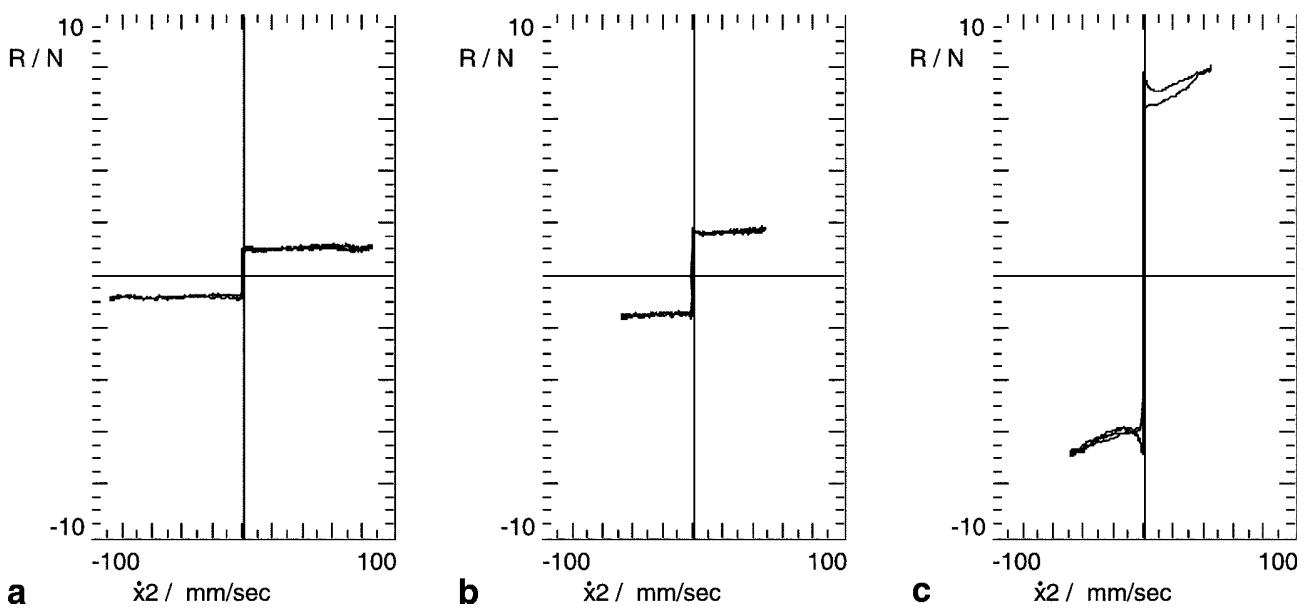

Fig. 9a-c. Friction characteristics for different normal forces 

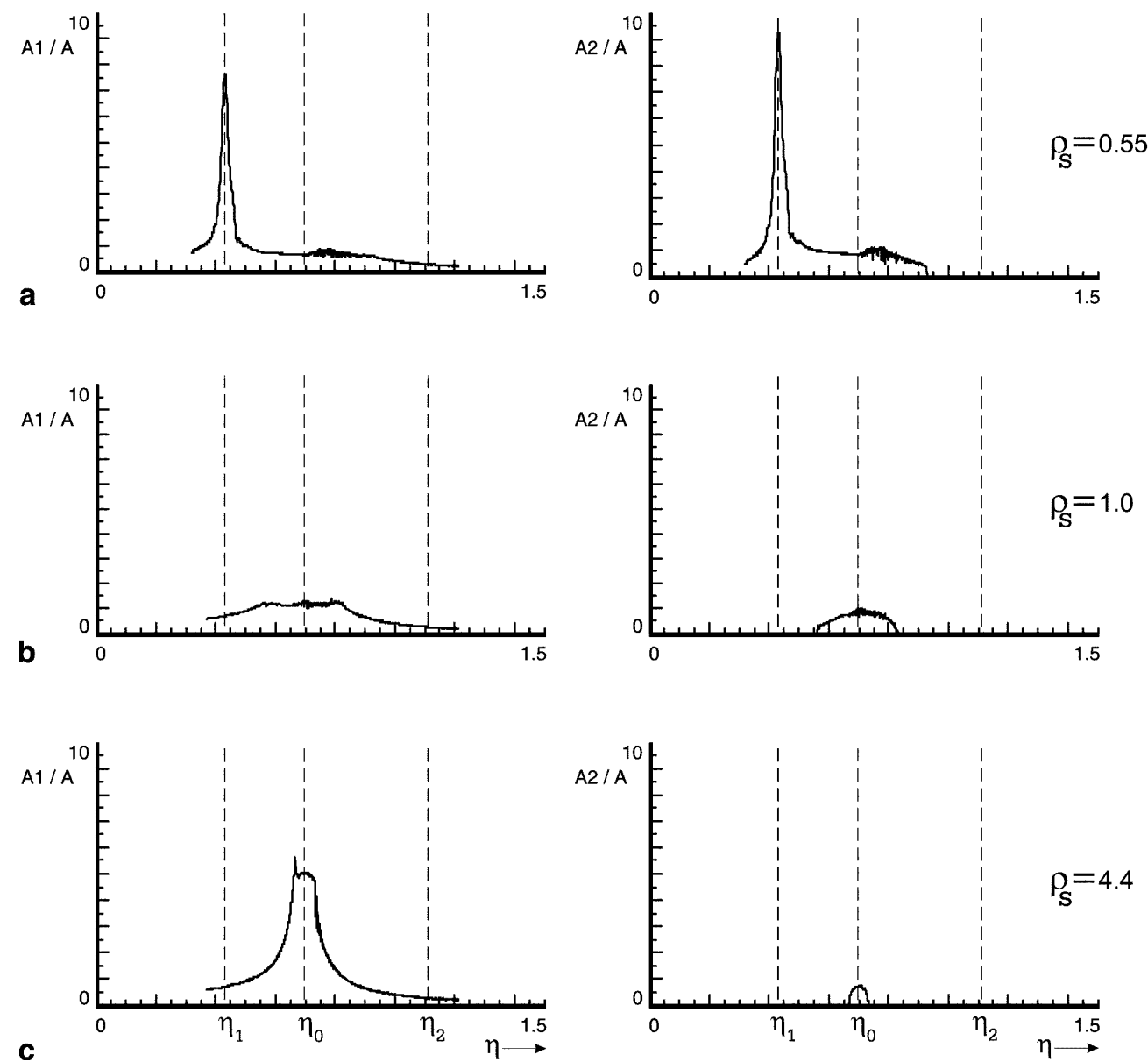

Fig. 10a-c. Frequency response curves for different normal forces

$\max \left(x_{i}\right)$ and minimum $\min \left(x_{i}\right)$ of the motions were searched to construct the ordinates $\left[\max \left(x_{i}\right)-\min \left(x_{i}\right)\right] / 2, i=1,2$ of the response curves.

Figure 10 shows the frequency response curves corresponding to the friction forces of Fig. 9. Both axes are made nondimensional in the same way as those of Figs. 3-5. This allows a quantitative comparison of the experimental results with those of the theory. The vertical dotted lines point out the frequencies $\eta_{0}, \eta_{1}$ and $\eta_{2}$, which agree with the theoretical ones.

Small friction, Fig. 10a, is identical to the plots in Figs. 3d, 4d and 5e. A properly adjusted normal force, Fig. 10b, eliminates all resonance peaks corresponding to Figs. $3 \mathrm{f}, 4 \mathrm{f}$ and $5 \mathrm{f}$. Large friction, Fig. 10c, leads to the resonance of the 1-DOF system discussed in Figs. 3g, $4 \mathrm{~g}$ and $5 \mathrm{~g}$.

Despite the fact of different characteristics for different normal forces, the results gained experimentally do not only show high consistency with the theoretically predicted ones in a phenomenological sense but also in the quantitative behaviour.

\section{5}

\section{Conclusions}

The effectiveness of a passive vibration absorber with dry friction mainly depends on the existence of stick phases at the friction device during motion. In addition, the displacement during the sliding phases must be made sufficiently large to eliminate mechanical energy from the system. These demands are basically controlled by the magnitude of the threshold value $\rho_{s}$ of the static friction force, which can be adjusted by the user. Despite the fact of harmonic excitation, the system becomes permanently detuned. Because of alternating degrees of freedom in each period of excitation, no definite resonance points can exist. The special shape of the dynamic friction laws during sliding, the so-called characteristic, has less influence, least of all on the saturation phenomenon. 
The possibility of detuning an oscillating mechanical system seems to be a general property of nonsmooth dynamic systems. A totally different problem shows the same effect. The resonance of a vibrating beam can be suppressed by adding a second beam with different natural frequencies. During motion both beams contact each other. The total system becomes detuned by a sequence of impacts, [12].

\section{References}

1. Ibrahim, R.A.: Friction-induced vibration, chatter, squeal, and chaos. Appl Mech Rev 47 (7) (1994) 209-253

2. Pfeiffer, F.; Glocker, Ch.: Multibody dynamics with unilateral contacts. J Wiley (1996)

3. Dormand, J.P.; Prince, P.J.: A family of embedded Runge-Kutta formulae. J Comput Appl Math 6 (1) (1980) 19-26

4. Meijaard, J.P.: Efficient numerical integration of the equations of motion of nonsmooth mechanical systems. Z Angew Math Mech 77 (6) (1997) 419-427

5. Vielsack, P.: Orbitale Stabilität nichtglatter Bewegungen bei permanenten numerischen Störungen. Z Angew Math Mech 79 (Suppl. 1) (1999) 105-108

6. Vielsack, P.; Hartung, A.: An example for the orbital stability of permanently disturbed nonsmooth motions. Z Angew Math Mech 79 (6) (1999) 389-397

7. Hartung, A.; Vielsack, P.: Non-unique periodic stick-slip motions of a forced 2-DOF-friction oscillator. Submitted to Z Angew Math Mech (Proceedings of the GAMM-Meeting Metz, 1999)

8. Queini, S.S.; Nayfeh, A.H.; Pratt, J.R.: A review of development and implementation of an active nonlinear vibration absorber. Arch Appl Mech 69 (1999) 585-620

9. Vielsack, P.: Stick-slip instability of decelerative sliding. Int Non-Linear Mech (accepted for publication)

10. Schmieg, H.; Vielsack, P.: Modellbildung und experimentelle Untersuchungen zum Bremsenquietschen. Z Angew Math Mech 78 (1998) 709-710

11. Van de Velde, F.; De Baets, P.: The relation between friction force and relative speed during the slip-phase of a stick-slip cycle. Wear 219 (1998) 220-226

12. Engleder, T.; Vielsack, P.; Spiess, H.: Damping by impacts, an application of nonsmooth dynamics. Proc of the Conf Nonlinear Oscill in Mech Syst (1998) 134-144 